\title{
POLARIZATION IN OPTICAL FIBERS
}

\author{
T.R. WoLIŃSKI* \\ Institute of Physics, Warsaw University of Technology \\ Koszykowa 75, 00-662 Warszawa, Poland
}

Physical origin of polarization phenomena in highly birefringent optical fibers including nonlinear optical effects is discussed and their impact on applications to polarimetric optical fibers sensors is underlined.

PACS numbers: 42.81.-i

\section{Introduction}

Optical fibers exhibit particular polarization properties. The guided electromagnetic fields in optical fiber waveguides are called inhomogeneous plane waves since their amplitudes are not stable within the plane wave and the fields are characterized, in most cases, by non-transverse components.

In the description of polarization phenomena in optical fibers there are generally two approaches [1]. The first one treats an optical fiber as an optical waveguide in which light being a kind of electromagnetic wave of optical frequencies can be guided in the form of waveguide modes. This approach identifies basic polarization eigenmodes of a fiber and relates them to the polarization state of the guided light. Changes in output polarization are described in terms of polarization-mode coupling due to birefringence changes acting as perturbations along the fiber.

Another approach treats an optical fiber like any other optical device which transmits light and the fiber can be divided into separated sections behaving like polarization state shifters. Here, polarization evolution in a fiber can be described by one of the three general formalisms: by the Jones vectors and matrices formalism, by the Stokes vectors and Mueller matrices formalism, or by the Poincaré sphere representation.

Since optical fibers allow very large propagation distances even very small birefringence effects can cumulate along fiber and their random distribution over the large lengths causes polarization properties of guided light generally difficult to determine.

Although polarization effects in optical fibers have initially played a minor role in the development of light-wav systems their importance is still growing. Before 1980 it was impossible to expivit the polarization modulation in a fiber for

*e-mail: wolinski@if.pw.edu.pl, http://www.if.pw.edu.pl./wolinski 
sensing applications since the conventional single-mode fibers manufactured for telecommunication use do not hold the optical wave amplitude in a particular polarization state. The appearance of highly-birefringent (Hi-Bi) polarization-maintaining (PM) fibers has created a novel generation of fiber-optic sensors known as polarimetric fiber sensors which utilize polarization (phase) modulation within these fibers or at their output due to various external perturbations describing the physical environment.

\section{Polarization phenomena in optical fibers}

\subsection{Polarization of the optical fiber modes}

The exact description of the modes propagating in fiber is complicated, since they are six hybrid-field components of great mathematical complexity.

The modes with the strong electric $E_{z}$ field compared to the magnetic $H_{z}$ field along the direction of propagation ( $z$ axis) are designated as EH modes. Similarly, those with a stronger $H_{z}$ field are called HE modes. These modes are hybrid since they consist of all six field components (3 electric and 3 magnetic) and possess no circular symmetry. The propagating modes are discrete and require two indexes $(l, p)$ to be identified: $\mathrm{HE}_{l p}, \mathrm{EH}_{l p}$.

For $l=0$, the hybrid modes are analogous to the transverse-electric (TE) and the transverse-magnetic (TM) modes of planar waveguides and there exist two linearly polarized sets of modes circularly symmetric with vanishing either the $E$ or $H$ longitudinal field components: $\mathrm{TE}_{0 p}\left(E_{z}=0\right)$ and $\mathrm{TM}_{0 p}\left(H_{z}=0\right)$. The lowest order transverse modes $\mathrm{TE}_{01}$ and $\mathrm{TM}_{01}$ have cutoff frequencies: $V=V_{\mathrm{c}}=2.405$, where $V$ is the normalized frequency defined as

$$
V=\frac{2 \pi}{\lambda} \cdot a \sqrt{n_{\mathrm{co}}^{2}-n_{\mathrm{cl}}^{2}}
$$

where $a$ is the core radius, $\lambda$ is the free space wavelength, $n_{\mathrm{co}}\left(n_{\mathrm{cl}}\right)$ is the refractive index of the fiber core (cladding), $\sqrt{n_{\mathrm{co}}^{2}-n_{\mathrm{cl}}^{2}}=N A$ is the numerical aperture of the fiber used in optics to express the ability of the system to gather the light.

The lowest order mode of a cylindrical waveguide is the $\mathrm{HE}_{11}$ mode which has zero cutoff frequency. The field distribution $E(r, t)$ corresponding to the $\mathrm{HE}_{11}$. mode has three nonzero components $E_{x}, E_{y}$, and $E_{z}$ (in Cartesian coordinates) and among these, either $E_{x}$ or $E_{y}$ dominates. However, even a single-mode fiber is not truly single-mode since the electric field of the $H_{11}$ mode has two polarizations orthogonal to each other that constitute two polarization modes of a single-spatial-mode fiber.

A significant simplification in the description of these modes is based on the fact that most fibers for practical applications use core materials whose refractive index is only very slightly higher than that of the surrounding cladding, i.e. $n_{\mathrm{co}}-n_{\mathrm{cl}} \ll 1$.

This assumption leads to the so-called "weakly-guiding approximation" in which instead of six-component field only four field components need to be considered. For a weakly-guiding fiber $\left(n_{\mathrm{co}} \approx n_{\mathrm{cl}}\right)$, there are approximate mode solutions defined as linearly polarized $\mathrm{LP}_{l p}$ modes of different azimuthal $(l)$ and radial $(p)$ mode numbers. The idea of LP modes was originally introduced by Gloge [2] who 
showed that for the lower-order modes, the combination modes have the electric. field configuration resembling a linearly polarized pattern.

The $\mathrm{LP}_{l p}$ modes count the group of modes appearing together as a single mode and they are an example of pseudo-modes with the property of changing their cross-sectional intensity and polarization pattern as the mode propagates. However, the $\mathrm{LP}_{l p}$ modes are superpositions of the true, generally hybrid, waveguide modes namely $\mathrm{HE}_{l+1, p}$ and $\mathrm{EH}_{l-1, p}$ modes.

In an isotropic fiber the modes of zero azimuthal order $(l=0)$ are twofold degenerate: two polarization modes $\mathrm{LP}_{0}^{x}$ and $\mathrm{LP}_{0 p}^{y}$ are possible, while modes of non-zero azimuthal order $(l>0)$ are fourfold degenerate: twofold orientational degeneracy (even and odd), and twofold polarization degeneracy ( $x$ and $y$ ). In this case four polarization modes, namely $\mathrm{LP}_{l p}^{x, \mathrm{e}}, \mathrm{LP}_{l p}^{y, \mathrm{e}}, \mathrm{LP}_{l p}^{x, \mathrm{o}}$, and $\mathrm{LP}_{l p}^{y, \circ}$ can be guided along the fiber.

In the isotropic case the single-mode fibers (normalized frequency parameter $V \leq 2.405$ ) with perfect circular cores support two degenerated orthogonal polarization modes $\mathrm{HE}_{11}^{x}$ and $\mathrm{HE}_{11}^{y}$ of the propagation mode $\mathrm{HE}_{11}$ with the same spatial intensity distribution, which is exactly the linearly polarized $L \mathrm{P}_{01}$ mode in the weakly guiding approximation. The fundamental or the lowest order $\mathrm{HE}_{11}$ mode, named after Gloge [2] the $\mathrm{LP}_{01}$ mode, exhibits an amplitude of a revolu-. tionary symmetry and may be linearly polarized either along the $x$ or the $y$ axis. Its two degenerated polarization modes $L P_{01}^{x}$ and $L P_{01}^{y}$ have the same propagation constant $\beta$ on a perfect cylindrically symmetric fiber. These polarization modes constitute a basis of two orthogonal and normalized states of linear polarizations and the electric fields of these modes are given by

$$
E_{x, y}=E_{0}\left\{\begin{array}{lc}
\frac{J_{0}(u r / a)}{J_{0}(u)}, & 0 \leq r<a, \\
\frac{K_{0}(u r / a)}{K_{0}(u)}, & r>a .
\end{array}\right.
$$

The normalization constant amplitude $E_{0}$ can be determined from the power relation [3]:

$$
E_{0}=\frac{w}{V} \frac{J_{0}(u)}{J_{1}(u)}\left(\frac{2 z_{0}}{\pi a^{2} n_{\mathrm{cl}}}\right)=\frac{u}{V} \frac{K_{0}(w)}{K_{1}(w)}\left(\frac{2 z_{0}}{\pi a^{2} n_{\mathrm{cl}}}\right),
$$

where $z_{0}=\omega u / k_{0}$ is the plane-wave impedance in a vacuum, $J_{l}$ and $K_{l}(l=0,1)$ are the Bessel functions of the first kind and the modified Bessel functions, respectively, and $\omega$ is the angular frequency corresponding to the free space wavelength $\lambda$.

The next four higher-order modes: $\mathrm{TE}_{01}, \mathrm{TM}_{01}, \mathrm{HE}_{21}^{\text {even }}$ and $\mathrm{HE}_{21}^{\text {odd }}$ modes $(2.405<V<3.832)$ have slightly different propagation velocities and almost the. same cross-sectional optical intensity distributions. In the weakly guiding approximation these four second-order modes become fourfold degenerate and are denoted as $\mathrm{LP}_{11}$ modes. The field distributions of four independent linear combinations of the waveguide modes: $\mathrm{TM}_{01}-\mathrm{HE}_{21}, \mathrm{TE}_{01}-\mathrm{HE}_{21}, \mathrm{TE}_{01}+\mathrm{HE}_{21}, \mathrm{TM}_{01}+\mathrm{HE}_{21}$ presented in Fig. 1 constitute the linearly polarized second-order $\left(\mathrm{LP}_{11}\right)$ modes as a single linear electric field vector. Fibers operating in this regime are two-mode (or bi-modal) fibers. In fact, the two-mode fiber supports six modes: two polarizations of the fundamental $L P_{01}$ mode and two polarizations of each of two lobe orientations (even and odd) of the second-order LP $P_{11}$ mode: $L P_{11}^{e}$ and $L P_{11}^{o}$. 

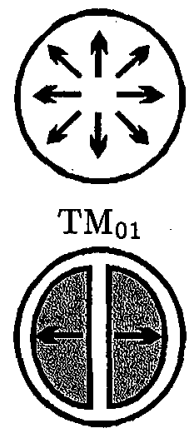

LP $P_{11}^{e x}$

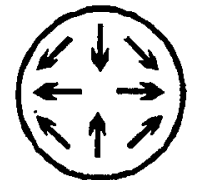

$\mathrm{HE}_{21}$

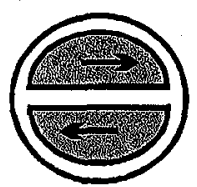

LP $P_{11}^{o x}$

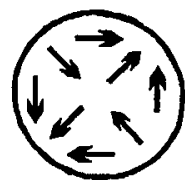

$\mathrm{HE}_{21}$

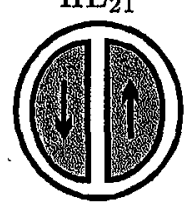

$\mathrm{LP}_{11}^{e y}$

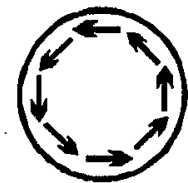

$\mathrm{TE}_{01}$

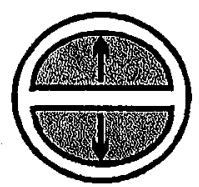

$\mathrm{LP}_{11}^{o y}$

$\mathrm{TM}_{01}-\mathrm{HE}_{21} \quad \mathrm{TE}_{01}+\mathrm{HE}_{21} \quad \mathrm{TE}_{01}-\mathrm{HE}_{21} \quad \mathrm{TM}_{01}+\mathrm{HE}_{21}$

Fig. 1. The electric field vector of the waveguide modes, the $\mathrm{TE}_{01}, \mathrm{TM}_{01}, \mathrm{HE}_{21}$ ( $\mathrm{t}$ wo polarizations) and their four independent linear contributions to the $\mathrm{LP}_{11}$ spatial mode.

\subsection{Optical fibers sensitive to polarization effects: Hi-Bi PM fibers}

An ideal isotropic fiber propagates any state of polarization launched into the fiber unchanged. However, the realization of the perfectly isotropic single-mode fiber bores huge manufacturing requirements as regards the ideal circularity of the core as well as lack of mechanical stress. Since in the ideal cylindrical fiber the fundamental $\mathrm{LP}_{01}$ mode contains two degenerated orthogonally polarized modes. they are propagating at the same phase velocity.

In real single-mode fibers which possess nonzero internal birefringence, both orthogonally polarized modes have randomly different phase velocities, causing fluctuations of the polarization state of the light guided in the fiber.

In highly-birefringent polarization-maintaining fibers, the difference between the phase velocities for the two orthogonally polarized modes is high enough to avoid coupling between these two modes. Fibers of these class have a built-in well-defined, high internal birefringence obtained by designing a core and/or cladding with noncircular (mostly elliptical) geometry, or by using anisotropic stress applying parts built into the cross-section of the fiber. Various types of $\mathrm{Hi}-\mathrm{Bi}$ polarization-maintaining fibers are presented in Fig. 2.
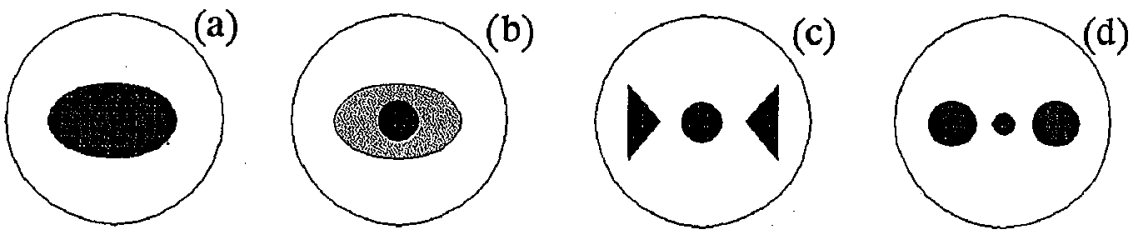

Fig. 2. Various types of $\mathrm{Hi}-\mathrm{Bi}$ polarization-maintaining fibers: (a) elliptical core, (b) elliptical internal cladding, (c) bow-tie, (d) PANDA. 
These include elliptical-core (a), stress-induced elliptical internal cladding (b), bow-tie (c) or PANDA (d) fibers. The magnitude of the internal birefringence is characterized by the beat length of the two polarization modes

$$
L_{\mathrm{B}}=2 \pi /\left|\beta_{y}-\beta_{x}\right|
$$

and is responsible for phase difference changes along the longitudinal $z$ axis of the Hi-Bi fiber. The spatial period of these changes reflects the changes in the polarization states along the fiber.

Since linearly birefringent (anisotropic) optical fibers have a pair of preferred orthogonal axes of symmetry (birefringence axes), two orthogonal quasilinear polarized field components $\mathrm{HE}_{11}^{x}$ and $\mathrm{HE}_{11}^{y}$ of the fundamental mode $\mathrm{HE}_{11}\left(\mathrm{LP}_{01}\right)$ which propagate for all values of frequency (wavelength) have electric fields that are polarized along one of these two birefringence axes. Hence, light polarized in a plane parallel to either axis will propagate without any change in its polarization but with different velocities. However, injection of any other input polarization excites both field components $\mathrm{HE}_{11}^{x}$ and $\mathrm{HE}_{11}^{y}$ and as these two orthogonal mode components are characterized by different propagation constants $\beta_{x}$ and $\beta_{y}$ (degeneracy of the fundamental mode is lifted) they run into and out of phase at a rate determined by the birefringence of the $\mathrm{Hi}-\mathrm{Bi}$ fiber producing at the same time a periodic variation in the transmitted polarization state from linear through elliptic to circular and.back again.

The relevant feature of Hi-Bi two-mode fibers is that only two second-order modes $\left(L P_{11}^{e}\right)$ propagate instead of four. This means that over a large region of the optical spectrum the two-mode $\mathrm{Hi}-\mathrm{Bi}$ fiber guides only four polarization modes: two orthogonal linearly polarized fundamental $L P_{01}^{x}, L P_{01}^{y}$ eigenmodes and the even second-order LP ${ }_{11}^{e x}, L P_{11}^{e y}$ spatial modes whose propagation constants we denote by $\beta_{0}^{x}, \beta_{0}^{y}, \beta_{1}^{x}, \beta_{1}^{y}$, instead of six as in case of isotropic fibers with perfect circular cores.

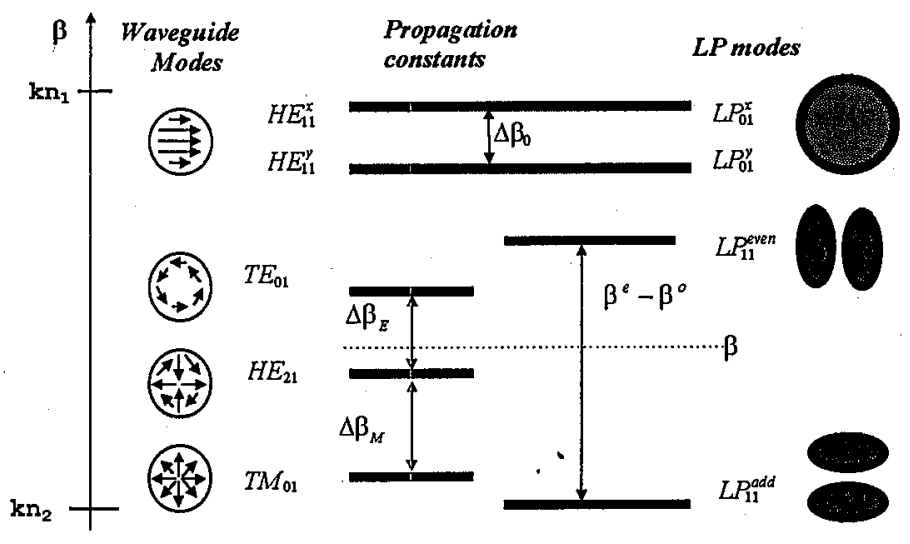

Fig. 3. Propagation constants for the lower-order waveguide and LP modes of a Hi-Bi fiber. 
Figure 3 presents propagation constants for the lower-order waveguide and LP modes of a linearly birefringent Hi-Bi fiber, where $\Delta \beta_{\mathrm{M}}=\beta_{\mathrm{TM}}-\beta_{\mathrm{HE}} ; \Delta \beta_{\mathrm{E}}=$ $\beta_{\mathrm{TE}}-\beta_{\mathrm{HE}}$, and $\beta=\left(\beta_{\mathrm{TM}}+2 \beta_{\mathrm{HE}}+\beta_{\mathrm{TE}}\right) / 4=\left(\beta^{\mathrm{e}}+\beta^{\circ}\right) / 2, \beta_{\mathrm{TM}}-\beta_{\mathrm{HE}}$, and $\Delta \beta_{0}=\beta_{0}^{x}-\beta_{0}^{y}$.

\subsection{The Jones formalism}

To describe quantitatively polarization transformation due to birefringence changes (intrinsic and induced) in Hi-Bi fiber a $2 \times 2$ unitary complex matrix the Jones matrix formalism [4] which is limited to the strictly monochromatic light sources - is applied

$$
\boldsymbol{E}^{\text {out }}(\lambda)=\boldsymbol{M}(V, \lambda) \cdot \boldsymbol{E}^{\text {in }}(\lambda),
$$

where $\lambda$ is the wavelength of propagating light, $M$ is propagation matrix depending on the physical environment represented by vector $\boldsymbol{V}$ and usually expressed as a product of three terms [5]

$$
M=T \exp (i \phi) J,
$$

where $T$ is the scalar transmittance, $\phi$ denotes the mean phase retardance and $\boldsymbol{J}$ is the birefringence (Jones) matrix of the fiber. The matrix becomes the identity matrix $I$ in case of an isotropic fiber with a perfect cylindrical symmetry.

For a linearly birefringent fiber

$$
\boldsymbol{J}=\boldsymbol{J}_{l}=\left[\begin{array}{cc}
\exp (\mathrm{i} \Delta \phi / 2) & 0 \\
0 & \exp (-\mathrm{i} \Delta \phi / 2)
\end{array}\right],
$$

where $\Delta \phi$ denotes linear relative phase retardance between the eigenmodes and fiber behaves like a simple linear retarder.

\section{Birefringence in optical fibers}

An ideal isotropic fiber has no birefringence. It propagates any state of polarization launched into the fiber unchanged.

Real fibers possess some amount of anisotropy owing to an accidental loss of circular symmetry. This loss is due to either a noncircular geometry of the fiber or a nonsymmetrical stress field in the fiber cross-section.

When birefringence is introduced into an isotropic fiber, the circular symmetry of the ideal fiber is broken thus producing the anisotropic refractive index distribution into the core region. The asymmetry results from either intrinsic birefringence including a geometrical deformation of the core and stresses induced during the manufacturing process or material anisotropy due to induced (extrinsic) elastic birefringence.

\subsection{Intrinsic birefringence}

Intrinsic birefringence is introduced in the manufacturing process and is a permanent feature of the fiber. It comprises any effect that causes a deviation from the perfect rotational symmetry of the ideal fiber. A noncircular (elliptical) core gives rise to geometrical (shape) birefringence, whereas a nonsymmetrical stress field in the fiber cross-section creates stress birefringence. Stress birefringence is induced by the photoelastic effect during the fiber manufacturing process. 
Asymmetrical lateral stresses may also be induced by surrounding the circular core by stress applying parts - zones having bow-tie shapes (bow-tie $\mathrm{Hi}-\mathrm{Bi}$ fibers) or two circles (PANDA Hi-Bi fibers).

\subsection{Induced (extrinsic) birefringence}

Birefringence can also be created whenever a fiber undergoes elastic stresses resulting from external perturbations such as hydrostatic pressure, longitudinal strain, squeezing, twisting, bending, etc. acting on the fiber from outside. The perturbation induced in the permittivity tensor through the photoelastic effect lifts. the degerveracy of the linearly polarized modes and induces extrinsic birefringence.

A number of important perturbations and the resulting induced birefringences include: internal stress anisotropy, external lateral force, bending, transverse electric field, elastic twist, axial magnetic field, axial strain, temperature, hydrostatic pressure and have been described elsewhere [6-10].

\subsection{Nonlinear birefringence}

Nonlinear or self-induced birefringence relies on the nonlinear coupling between the orthogonally polarized components of an optical wave that changes the refractive index by different amounts of $\Delta n_{x}$ and $\Delta n_{y}$ due to nonlinear contributions [11]

$$
\Delta n_{x}=n_{2}\left[\left|E_{x}\right|^{2}+\frac{2}{3}\left|E_{y}\right|^{2}\right], \quad \Delta n_{y}=n_{2}\left[\left|E_{y}\right|^{2}+\frac{2}{3}\left|E_{x}\right|^{2}\right],
$$

where $n_{2}$ is the nonlinear-index coefficient given by the formula

$$
n_{2}=\frac{3}{8 n} \operatorname{Re}\left(\chi_{\chi \chi \chi \chi}^{(3)}\right)
$$

and defined by one component of the 4-rank nonlinear susceptibility tensor $\chi_{\chi \chi \chi \chi \chi}^{(3)}$.

In the case of silica fibers, where the dominant nonlinear contribution is of electronic origin, the nonlinear-index coefficient has a value $1.2 \times 10^{-22} \mathrm{~m}^{2} / \mathrm{V}$ or $3.2 \times 10^{-22} \mathrm{~m}^{2} / \mathrm{W}$.

As the wave propagates along the fiber, it acquires an intensity-dependent nonlinear phase given by

$$
\begin{aligned}
& \phi_{x}^{\mathrm{nl}}=\frac{2 \pi}{\lambda} L \Delta n_{x}=\frac{2 \pi}{\lambda} L n_{2}\left[\left|E_{x}\right|^{2}+\frac{2}{3}\left|E_{y}\right|^{2}\right], \\
& \phi_{y}^{\mathrm{nl}}=\frac{2 \pi}{\lambda} L \Delta n_{y}=\frac{2 \pi}{\lambda} L n_{2}\left[\left|E_{y}\right|^{2}+\frac{2}{3}\left|E_{x}\right|^{2}\right] .
\end{aligned}
$$

The first term in the brackets is responsible for self-phase modulation (SPM), while the second term results from the phase modulation of one polarization (wave) by the copropagating orthogonal polarization (wave) and is responsible for the so-called cross-phase modulation (XPM). The XPM-induced nonlinear coupling between the field components $E_{x}$ and $E_{y}$ creates nonlinear birefringence that changes the state of polarization (SOP) if the input light is elliptically polarized.

The nonlinear coupling between the two orthogonally polarized components of the optical wave is referred as nonlinear or self-induced birefringence and has. many device applications. 


\subsubsection{Optical Kerr effect}

The optical Kerr effect involves transmission changes of a weak probe beam due to nonlinear birefringence induced by a strong pump beam. In all-fiber realization both pump and probe beams are linearly polarized at $45^{\circ}$ to each other at the fiber input. A crossed polarizer blocks the fiber output in the absence of the pump beam. The presence of the pump modifies the phase difference between orthogonal components of the probe at the output of a fiber of length $L$

$$
\Delta \phi=\Delta \phi^{1}+\Delta \phi^{n !}=\frac{2 \pi}{\lambda} L\left[\Delta n^{1}+n_{\mathrm{K}}\left|E_{\mathrm{pump}}\right|^{2}\right],
$$

where $\Delta n^{1}=n_{x}-n_{y}$ is the linear birefringence of the fiber, $n_{\mathrm{K}}$ is the Kerr coefficient, and $E_{\text {pump }}$ is the pump intensity.

\subsubsection{Pulse shaping}

Nonlinear birefringence induced by a pulse can be used to change its own shape. Here, the signal itself produces the nonlinear birefringence and modifies its own SOP. If an input beam is polarized in such a way that it excites both orthogonal polarizations, the field components $E_{x}$ and $E_{y}$ change the refractive indices $n_{x}$ and $n_{y}$ by the amount $\Delta n_{x}$ and $\Delta n_{y}$, see Eq. (8). Hence the resulting phase shift at the fiber output is given by

$$
\Delta \phi^{\mathrm{nl}}=\frac{2 \pi L}{\lambda} \frac{n_{2}}{3}\left[\left|E_{y}\right|^{2}-\left|E_{y}\right|^{2}\right]
$$

and the transmitted power depends on input polarization angle and input power.

Generally, an accurate description of the polarization effects in $\mathrm{Hi}-\mathrm{Bi}$ fibers requires simultaneous consideration of both intrinsic linear birefringence and induced nonlinear birefringence effects. One of the most spectacular effects is the so-called polarization instability which manifests as large changes in the output SOP when the input power or input SOP are changed slightly. When the input beam is polarized close to the slow axis, the nonlinear birefringence adds to the intrinsic birefringence and the fiber is more birefringent. However, when the input beam is polarized close to the fast axis, the nonlinear birefringence decreases the intrinsic birefringence. When the input power is close to the critical power defined by

$$
P_{\mathrm{cr}}=\frac{3}{2} \frac{\Delta \beta}{\gamma}
$$

where $\Delta \beta$ is the intrinsic (linear) birefringence and $\gamma$ is a nonlinear parameter, the effective beat length becomes infinite (intrinsic birefringence vanishes). Further increase in the input power makes the fiber again birefringent but the roles of the slow and fast axes are reversed. Hence any slight changes in the input power close to the critical power cause large changes in the output SOP.

The polarization instability first observed in 1986 by Trillo et al. [12] proves that both birefringence axes (slow and fast) of a Hi-Bi PM fiber are not entirely equivalent. 


\section{Polarimetric fiber optic sensors}

The linearly polarization eigenmodes of Hi-Bi fiber are associated with phase retardation $\phi_{\mathrm{f}}$ and $\phi_{\mathrm{s}}$ where subscripts $\mathrm{f}$ and $\mathrm{s}$ stand for fast and slow azimuths, respectively. The total relative phase retardation between the two perpendicularly polarized eigenmodes propagating in $\mathrm{Hi}$-Bi fiber of a length $L$ can be expressed as

$$
\Delta \phi=\phi_{\mathrm{f}}-\phi_{\mathrm{s}}=(2 \pi / \lambda) \Delta n L,
$$

where $\lambda$ is the wavelength of the light and $\Delta n$ equal to $n_{\mathrm{f}}-n_{\mathrm{s}}$ is the difference between the effective indices of the polarization modes. This phase retardation can be easily changed by external factors (pressure, temperature, different stresses, etc.) and it creates the background of polarimetric fiber optic sensors (see Fig. 4).

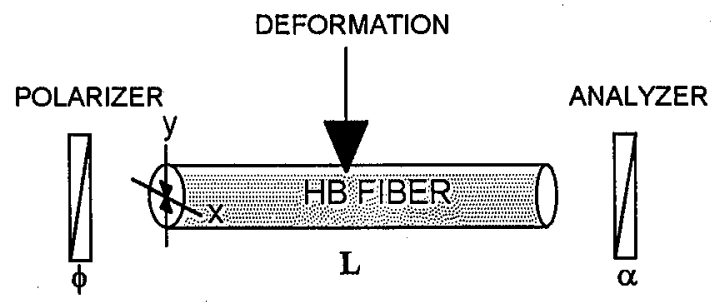

Fig. 4. Deformation effect in a Hi-Bi fiber modulates light intensity after the analyzer: $I(z=L)=(1 / 2)\left[1+\cos 2 \alpha \cos 2 \varphi+\left|\gamma_{i}\right| \sin \alpha \sin 2 \varphi \cos \Delta \Phi_{i}\right]$, where $\Delta \Phi_{i}$ signifies the differential phase of the light exiting the $\mathrm{Hi}-\mathrm{Bi}$ fiber and $\gamma_{i}$ is a mutual correlation function.

If quasimonochromatic light linearly polarized at an angle $\varphi$ with respect to the fiber's $x$ axis is launched into the fiber and an analyzer turned to an angle $\alpha$ is placed at the output of the fiber then the optical intensity detected will be

$$
I=\frac{1}{2}\left[1+\cos 2 \alpha \cos 2 \varphi+\left|\gamma_{0}\right| \sin 2 \alpha \sin 2 \varphi \cos \Phi_{0}\right],
$$

$\Phi_{0}=\Delta \beta_{0}^{0} L$ is the phase. The same dependence is valid for the $L P_{11}^{x}$ to $L P_{11}^{y}$ polarimetric interference but $\Delta \beta_{1}^{0}$ should be substituted instead of $\Delta \beta_{0}^{0}$.

When external perturbations are introduced, they lead to changes in the phase $\Phi_{0}=\Delta \beta_{0}^{0} L$ of the fundamental LP ${ }_{01}$ mode (or correspondingly $\Phi_{1}=\Delta \beta_{1}^{0} L$ for the $\mathrm{LP}_{11}$ ). Consequently, it will lead to a cosine variation of the observed intensity $I$ measured after the analyzer and that is in fact the polarization interference. The setup is then a polarimetric sensor [13-15]. The interfering beams in this case are the $L P_{01}^{x}$ and the $L P_{01}^{y}$ polarization modes. An input polarizer (if the light is not linearly polarized) acts as a splitter and the analyzer acts as a recombiner.

\subsection{Polarimetric smart structures}

There is at present much effort to apply polarization effects in optical fibers to measure strain in aircrafts or concrete structures using the concept of the so-called smart skins and structures. Smart structures and smart skins are structural components (particularly presumed for advanced aircrafts and space vehicles) with 
networks of fiber optic sensors directly embedded within their composite material matrices, which are valued by the aerospace industry for their light weight and high strength. The composite material are made with epoxies or polyimides. Structurally integrated polarimetric optical fiber sensors have emerged as an important part of sensors for smart structures applications. A more ambitious and - complex use of smart structures involves linking fiber optic sensors with real-time computer-control system aboard advanced aircraft. In this model, fiber-optic sensors are embedded in a panel to be integrated with the wing. The sensors monitor environmental effects, such as strain and bending, around as well as within the panel. In smart structures, fiber optic sensor become a part of the wing itself, and are of course not affected by electrical disturbance. In response to computer output, a fiber optic link could drive remote actuators. A complete smart structure would not only detect problems but respond to them instantaneously.

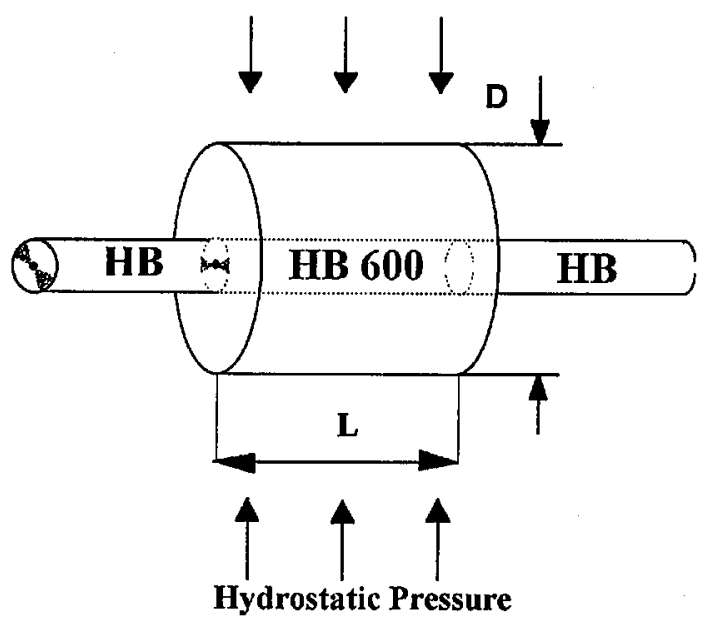

Fig. 5. The Hi-Bi fiber embedded in the cylindrical epoxy structure. The lead-in and lead-out $\mathrm{Hi}-\mathrm{Bi} 600$ fibers are spliced at different angles.

Very recently [16], a model of a polarimetric smart structure composed of the $\mathrm{Hi}-\mathrm{Bi}$ bow-tie fiber embedded in an epoxy cylinder has been demonstrated. The Hi-Bi fiber-based structure has been subjected to selected deformation effects mostly induced by hydrostatic pressure (up to $300 \mathrm{MPa}$ ) and temperature, whereas polarization properties of the transmitted optical signal have been investigated. The test samples were composed of the separated Hi-Bi fibers, as well as the same Hi-Bi fibers embedded in epoxy structures (Fig. 5). It appeared that the presence of the epoxy coating modifies the output characteristics of the Hi-Bi fiber (mostly due to elastic properties of the structure) as well as the level of the induced birefringence of the Hi-Bi fiber and hence influences polarization properties of the light propagating in the fiber.

The smart structures hydrostatic pressure characteristics has a fundamental period $T_{\mathrm{p}} \approx 100 \mathrm{MPa}$, which is twice as large as the period of the separated 


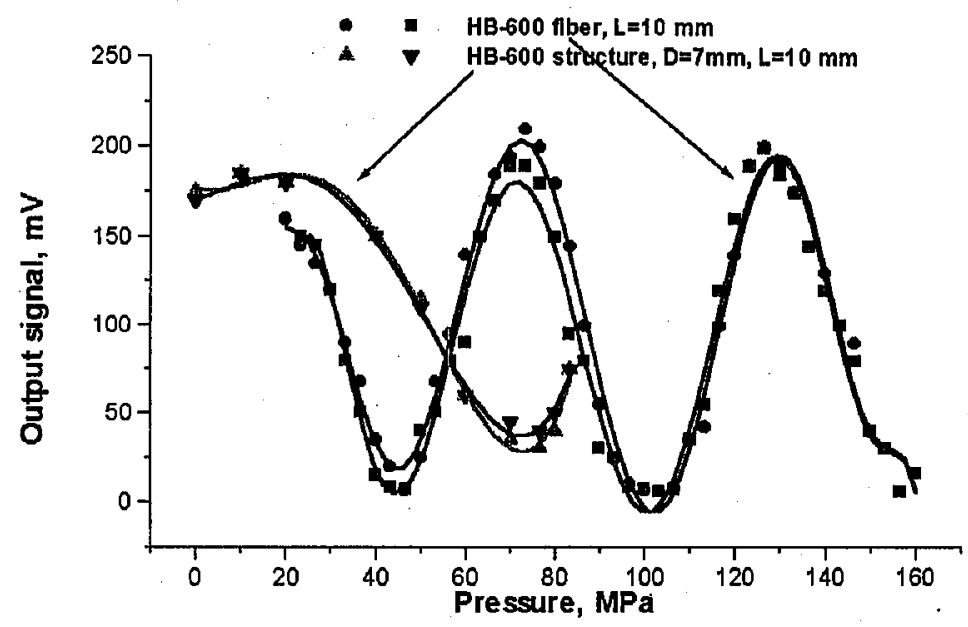

Fig. 6. Comparison of the pressure characteristics of the separated Hi-Bi fiber and the Hi-Bi fiber-based polarimetric smart structure.

Hi-Bi-600 bow-tie fiber $\left(T_{\mathrm{p}} \approx 50 \mathrm{MPa}\right)$, see Fig. 6 . Hence, the presence of the epoxy structure significantly modifies the output characteristics of the Hi-Bi fiber. As a consequence, the induced birefringence of the embedded Hi-Bi fiber changes less drastically than for the separated $\mathrm{Hi}-\mathrm{Bi}$ fiber probably to the fact that pressure induced stresses in the structure are separated between both the epoxy coating and the embedded Hi-Bi fiber.

\section{Conclusions}

The polarization effects in optical fibers have created a background for a novel generation of powerful and mostly sensing-oriented technique. These effects are inherently connected with intrinsic and induced birefringences of optical fibers, both in linear and nonlinear regimes of operation. The idea of polarization controlling of the guided optical field is the important issue and can be successfully realized only in the Hi-Bi fibers.

Identification of all the polarization phenomena existing in the Hi-Bi fibers opens up new perspectives on basic physical effects occurring in optical fiber waveguides and simultaneously creates new opportunities for smart sensing applications holding still great potential for optical fiber telecommunications.

\section{Acknowledgments}

This work was partially supported by the Warsaw University of Technology.

\section{References}

[1] C. Tsao, Optical Fibre Waveguide Analysis, Oxford University Press, Oxford 1992.

[2] D. Gloge, Appl. Opt. 10, 2252 (1971).

[3] C. Yeh, IEEE Trans. Educ. E-30, 43 (1987).

[4] R.C. Jones, J. Opt. Soc. Am. 31, 488 (1941). 
[5] Optical Fiber Sensors, Vol. 1,2, Eds. B. Culshaw, J.P. Dakin, Artech House, Boston 1989.

[6] W.J. Bock, A.W. Domański, T.R. Wolinski, Appl. Opt. 29, 3484 (1990).

[7] W.J. Bock, T.R. Woliński, Opt. Lett. 15, 1434 (1990).

[8] W.J. Bock, T.R. Woliński, T.A. Eftimov, Pure Appl. Opt. 5, 125 (1996).

[9] Fiber Optic Sensors - An Introduction for Engineers and Scientists, Ed. E. Udd, Wiley, New York 1991.

[10] R. Ulrich, Polarization and Birefringence Effects, in: Optical Fiber Rotation Sensing, Ed. W.K. Burns, Academic Press, San Diego 1994, Ch. 2, p. 31.

[11] G.P. Agarwal, Nonlinear Fiber Optics, 2nd ed., Academic, San Diego 1995.

[12] S. Trillo, S. Wabnitz, R.H. Stolen, G. Assanto, C.T. Seaton, G. Stegemann, Appl. Phys. Lett. 49, 1224 (1986).

[13] I.P. Kaminov, IEEE J. Quantum Electron. QE-17, 15 (1981).

[14] S.C. Rashleigh, J. Lightwave Tech. LT-1, 312 (1983).

[15] T.A. Eftimov, J. Mod. Opt. 42, 541 (1995).

[16] T.R. Woliński, W. Konopka, A.W. Domański, Proc. SPIE 3475, 421 (1998). 